\title{
Comparative wood anatomy of species of Psychotria L. (Rubiaceae) in Atlantic Rainforest remnants of Rio de Janeiro State, Brazil
}

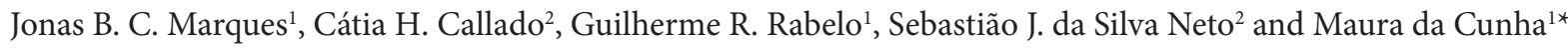

Received: January 10, 2015. Accepted: May 21, 2015

\begin{abstract}
Psychotria is the largest Rubiaceae genus and one of the most abundant in the Atlantic Rainforest of Rio de Janeiro State. The present work aims to characterize and compare the wood of nine of these species. The Psychotria wood were characterized by: slightly distinct growth rings; diffuse porosity; solitary vessels or on radial multiples of 2-6 or clusters of 3-5 vessel elements, with terminal and lateral simple perforation plates and vestured and alternate intervessel and vessel-ray pits; septate fiber-tracheids; and rare axial parenchyma. Although, the wood anatomy of the Psychotria may be considered homogeneous, the statistical analyses, based on qualitative and quantitative features, allowed the segregation of the species and indicated the importance of habit (arboreous or shrubby) on the wood anatomy of the species. It is worth mentioning that the sampling was realized in only one studied site (Ilha Grande), which contributed to the conclusion that the wood characteristics are more related to the specificities of each studied taxon than to with environmental variations.
\end{abstract}

Keywords: Ilha Grande, Psychotriae, Rubioideae, Tropical Forest, wood diagnostic characters

\section{Introduction}

The remaining remnants of Atlantic Forest in the state of Rio de Janeiro represent almost $20 \%$ of its original area in the state. One of the largest fragments is the island of Ilha Grande, the third largest Brazilian island and the largest in the state of Rio de Janeiro $\left(190 \mathrm{~km}^{2}\right)$. Being one of the largest remaining tracks of Atlantic Forest, and comprised predominantly of Dense Ombrophyllous Forest, the forest of Ilha Grande is of particular value for scientific study (Araujo \& Oliveira 1988; Oliveira \& Coelho Netto 2001; Callado et al. 2009).

Rubiaceae is one of the most important plant families, in terms of species richness, in the Atlantic Forest remnants of the state of Rio de Janeiro (Silva Neto \& Peixoto 2012). Psychotria is the largest genus of the Rubiaceae with approximately 2000 species (Davis et al. 2001). The genus is subdivided into three subgenera: Psychotria (pantropical), Tetramerae (some species in Africa and Madagascar) and Heteropsychotria (neotropical) based on morphological characters and geographical distribution (Steyermark 1972; Lopes et al. 2004). This genus is now considered paraphyletic and is found in both hemispheres in predominantly shady locations and in relatively moist soils in sub-woods (Taylor 1996; Bremer \& Eriksson 2009).

Among the 19 species of Psychotria that occur in Dense Ombrophyllous Forest of Rio de Janeiro (List of Species of the Brazilian Flora 2015), 16 occur on Ilha Grande, including nine woody species (Manão 2011).

The taxonomy of Psychotria is complex, due not only to the large number of species but also to the absence of morphological characters available for defining taxa (Nepokroeff et al. 1999). Authors such as Koek-Noorman (1969a; b), Koek-Noorman \& Hogeweg (1974), Rogers (1981; 1984), Ter Welle et al. (1983), Koek-Noorman \& Puff (1983; 1991), and Jansen et al. $(1997 ; 2002)$ have characterized the wood anatomy of Rubiaceae, examining its taxonomic, ecological and evolutionary aspects among the subfamilies, tribes, genera and species, and recognized the importance of wood anatomy for the taxonomic classification of this group. Koek-Noorman (1977) classified the wood of Rubiaceae as of two types, with type II characteristic of Rubioideae: solitary vessels, radial clusters of few vessel elements, septate libriform fibers and axial parenchyma absent or extremely rare.

This study aims to characterize the wood anatomy of nine species of Psychotria from the Atlantic Forest, in order to determine which characteristics are most useful for taxonomic study and which are more useful for ecological investigation. Ultimately this study aims to increase the understanding of anatomical features of this genus in order to better understand the adaptive strategies of its species.

\footnotetext{
${ }^{1}$ Setor de Biologia Vegetal, Laboratório de Biologia Celular e Tecidual, Universidade Estadual do Norte Fluminense Darcy Ribeiro, 28013-602, Campos dos Goytacazes, RJ, Brazil

${ }^{2}$ Departamento de Biologia Vegetal, Instituto de Biologia Roberto Alcantara Gomes, Universidade do Estado do Rio de Janeiro, 20550-900 Rio de Janeiro, RJ, Brazil * Corresponding author: maurauenf@gmail.com
} 


\section{Materials and Methods}

Sampling was performed on the southeast side of Ilha Grande, Rio de Janeiro State, Brazil, (2310’45”S, $23^{\circ} 11^{\prime} 24^{\prime \prime}$ S, $44^{\circ} 11^{\prime} 01^{\prime \prime} \mathrm{W}, 44^{\circ} 11^{\prime} 47^{\prime \prime} \mathrm{W}$ ) in an area of submontane Dense Ombrophyllous Forest of the Atlantic Forest biome. Five individuals of each of the following species were sampled: Psychotria hoffmannseggiana Müll. Arg., Psychotria brasiliensis Vell., Psychotria carthagenensis Jacq., Psychotria deflexa DC., Psychotria leiocarpa Cham. \& Schlecht., Psychotria nuda Cham. \& Schlecht., Psychotria pubigera Schltdl., Psychotria ruelliifolia Cham. \& Schltdl., and Psychotria vellosiana Benth. (Tab. 1).

Wood samples were obtained by non-destructive methods. For arboreous specimens samples were taken using a Pressler probe at approximately $1.30 \mathrm{~m}$ above the ground, whereas for shrubby specimens samples were taken as stem disks using a machete. Part of the wood samples and permanent slides were deposited and registered in the wood collection of the Universidade Estadual do Norte Fluminense (UENF), whereas the remainder was used for anatomical identification.

Blocks were cut from each sample and 15-20 $\mu \mathrm{m}$ sections were made with a Leica SM 2010R sliding microtome (Nussloch, Germany) in transverse and longitudinal (radial and tangential) planes. The sectioned material was dehydrated in ethanol and double stained with Astra Blue and hydroalcoholic Safranin (Burger \& Richter 1991). Macerations were prepared using Jeffrey solution (Johansen 1940), and stained with aqueous Safranin. All anatomical measurements were performed using a light microscope (Axioplan Zeiss, Oberkochen, Germany). Vulnerability (IV = tangential diameter of vessels/vessel frequency) and mesomorphy (IM = IV X length of vessel elements) were calculated following the method proposed by Carlquist (2001) (Tab. 1). In general, the measurement techniques and terminology follow IAWA guidelines (IAWA committee 1989).

For the scanning electron microscopy (SEM), material was prepared according to the methods of Jansen et al.
(2008), and observations made using a ZEISS-DSEM 962 (Oberkochen, Germany) scanning electron microscope.

Statistica 7.0 Software (StatSoft 1993, Tulsa, Oklahoma, USA) and PAST version 2.01 (Hammer et al. 2001) for Windows XP/Vista were used to perform the statistical analyses. The Shapiro-Wilks W test was used to evaluate the normality of each parameter (Zar 1996).

Qualitative analysis was performed on the nine species of Psychotria L., as well as Coussarea friburguensis M. Gomes., and Coussarea congestiflora Mull. Arg., as outgroup taxa, for which the wood anatomy had previously been described by Barros et al. (2001). For the qualitative analysis we created a binary matrix of presence and absence of wood anatomical characteristics, based on IAWA 1989, that differed among species. To ensure the segregation of species regardless of habit the analysis was performed excluding the variables of size, width and frequency of vessels. Cluster analysis and principal component analysis (PCA) were conducted using this matrix using the unweighted pair group method of analysis (UPGMA) (Sokal 1986).

To perform a quantitative analysis, fifteen wood anatomical features of the nine species shown in Tab. 2 were submitted to principal component analysis (PCA) (Sokal 1986). The Kruskal-Wallis test for nonparametric measurements was used to test the differences between the medians of each parameter analyzed among species.

\section{Results}

Among species comparisons of wood anatomy described below following the terminology of IAWA guidelines (IAWA committee 1989). The figures were from agreement with the rules and selected to present the most representative differences between characters and species. Figure 1 shows transversal (Fig. 1A-C), tangential (Fig. 1D-F) and radial sections (Fig. 1G-I). Figure 2 shows fibers (Fig. 2A-B), vessels (Fig. 2C-F) and ray cells (Fig. 2G). Figure 3 shows details of vessels (Fig. 3A-C), fibers (Fig. 3D) and ray cells (Fig. 3E-F).

Table 1. Characteristics of Psychotria species studied. $\mathrm{N}^{\circ}$ Reg. $=$ registration number in the UENF Wood collection, $\mathrm{DBH}=$ diameter at breast height in centimeters, $\mathrm{H}=$ height in meters, $\mathrm{VL}=$ vulnerability index, $\mathrm{ME}=$ mesomorphy index.

\begin{tabular}{|c|c|c|c|c|c|c|}
\hline Species & $\mathrm{N}^{\circ}$ Reg. & Habit & $\mathrm{DBH}$ & $\mathrm{H}$ & VL & $\mathrm{ME}$ \\
\hline P. hoffmannseggiana & $112,113,155,156$ e 157 & Shrubby & $4.2( \pm 1.09)$ & $1.8( \pm 0.44)$ & 0.127 & 141.4 \\
\hline P. brasiliensis & $134,135,136,137$ e 138 & Arborecent & $11.8( \pm 2.04)$ & $6.4( \pm 1.51)$ & 0.592 & 678.3 \\
\hline P. carthagenensis & $144,145,146,147$ e 148 & Arborecent & $18.4( \pm 2.88)$ & $12.8( \pm 2.16)$ & 2.172 & 2115.9 \\
\hline P. ruelliifolia & $107,115,152,153$ e 154 & Shrubby & $6.4( \pm 1.14)$ & $2.3( \pm 0.27)$ & 0.385 & 344.7 \\
\hline P. deflexa & $110,111,159,160$ e 161 & Shrubby & $4.6( \pm 1.14)$ & $2.3( \pm 0.57)$ & 0.156 & 125.5 \\
\hline P. leiocarpa & $106,114,158,162$ e 163 & Shrubby & $5.6( \pm 1.34)$ & $1.9( \pm 0.41)$ & 0.136 & 96.3 \\
\hline P. nuda & $108,130,131,132$ e 133 & Arborecent & $9.8( \pm 1.48)$ & $5.6( \pm 1.51)$ & 0.148 & 132.9 \\
\hline P. pubigera & $109,116,149,150$ e 151 & Arborecent & $7( \pm 1.87)$ & $6( \pm 1.58)$ & 0.315 & 307.6 \\
\hline P. vellosiana & $139,140,141,142$, e 143 & Arborecent & $18.8( \pm 2.58)$ & $10( \pm 2.12)$ & 2.653 & 2318.7 \\
\hline
\end{tabular}


Table 2. Quantitative wood anatomical features of the nine Psychotria studied. bar $=$ P. hoffmannseggiana; bra $=$ P. brasiliensis; car $=P$. carthagenensis; rue $=$ P. ruelliifolia; def $=$ P. deflexa; le $=P$. leiocarpa; $n u d=P . n u d a ; p u b=P$. pubigera; vell $=P$. vellosiana. $\mathrm{Q} 25=$ quartile $25, \mu=$ medians, $\mathrm{Q} 75=$ quartile 75 , $\mathrm{KW}=$ Kruskal-Wallis test between the medians.

\begin{tabular}{|c|c|c|c|c|c|c|c|c|c|c|c|}
\hline & Species & & bar & bra & car & rue & def & lei & nud & pub & vel \\
\hline \multirow{28}{*}{$\begin{array}{l}\infty \\
\tilde{\infty} \\
\infty \\
\infty\end{array}$} & \multirow{4}{*}{$\begin{array}{l}\text { Vessels } / \mathrm{mm}^{2} \\
\text { (N 50) }\end{array}$} & Q25 & 175 & 59 & 24 & 80 & 130 & 161 & 152 & 95 & 25 \\
\hline & & $\mu$ & 181 & 66 & 26 & 91 & 141 & 178 & 178 & 102 & 27 \\
\hline & & Q75 & 189 & 75 & 28 & 98 & 152 & 193 & 190 & 114 & 30 \\
\hline & & KW & $\mathrm{a}$ & be & $\mathrm{d}$ & $\mathrm{b}$ & c & $\mathrm{a}$ & $\mathrm{ac}$ & $\mathrm{bc}$ & de \\
\hline & \multirow{4}{*}{$\begin{array}{l}\text { Length }(\mu \mathrm{m}) \\
\quad(\mathrm{N} 125)\end{array}$} & Q25 & 931 & 1048 & 909 & 783 & 710 & 620 & 784 & 840 & 782 \\
\hline & & $\mu$ & 1120 & 1141 & 965 & 888 & 772 & 734 & 921 & 956 & 815 \\
\hline & & Q75 & 1245 & 1277 & 1000 & 984 & 838 & 789 & 993 & 1068 & 887 \\
\hline & & KW & $\mathrm{a}$ & $\mathrm{a}$ & $\mathrm{d}$ & be & c & c & bde & bd & $\mathrm{e}$ \\
\hline & \multirow{4}{*}{$\begin{array}{c}\text { Tang. Diameter }(\mu \mathrm{m}) \\
(\mathrm{N} 125)\end{array}$} & Q25 & 19.4 & 32.2 & 48.1 & 28.4 & 20.4 & 21.4 & 22.3 & 28.5 & 60.5 \\
\hline & & $\mu$ & 28.0 & 36.8 & 53.6 & 33.2 & 26.2 & 24.0 & 24.9 & 32.9 & 67.8 \\
\hline & & Q75 & 34.6 & 41.0 & 60.8 & 38.5 & 28.0 & 25.8 & 28.4 & 35.8 & 74.7 \\
\hline & & KW & $\mathrm{a}$ & $\mathrm{b}$ & $\mathrm{d}$ & $\mathrm{b}$ & $\mathrm{a}$ & $\mathrm{ac}$ & c & $\mathrm{b}$ & $\mathrm{d}$ \\
\hline & \multirow{4}{*}{$\begin{array}{l}\text { Rad. diameter }(\mu \mathrm{m}) \\
(\mathrm{N} 125)\end{array}$} & Q25 & 22.3 & 32.0 & 60.5 & 33.0 & 24.0 & 25.8 & 24.0 & 26.7 & 62.2 \\
\hline & & $\mu$ & 25.1 & 36.5 & 65.8 & 37.3 & 27.6 & 27.7 & 26.7 & 29.4 & 71.1 \\
\hline & & Q75 & 28.5 & 43.2 & 71.1 & 41.8 & 30.2 & 30.4 & 30.2 & 32.9 & 79.2 \\
\hline & & KW & $\mathrm{a}$ & b & $\mathrm{d}$ & b & $\mathrm{ac}$ & $\mathrm{ac}$ & ac & c & $\mathrm{d}$ \\
\hline & \multirow{4}{*}{$\begin{array}{l}\text { Wall thickness }(\mu \mathrm{m}) \\
\text { (N 125) }\end{array}$} & Q25 & 2.5 & 1.8 & 3.2 & 2.5 & 2.6 & 2.7 & 1.8 & 2.1 & 1.6 \\
\hline & & $\mu$ & 2.8 & 2.0 & 3.6 & 2.9 & 3.0 & 3.0 & 2.1 & 2.4 & 1.7 \\
\hline & & Q75 & 3.2 & 2.5 & 4.0 & 3.2 & 3.4 & 3.4 & 2.5 & 2.7 & 2.0 \\
\hline & & KW & $\mathrm{a}$ & b & c & $\mathrm{a}$ & $\mathrm{a}$ & $\mathrm{a}$ & b & b & $\mathrm{d}$ \\
\hline & \multirow{4}{*}{$\begin{array}{l}\text { Intervessel pits }(\mu \mathrm{m}) \\
(\mathbf{N} 125)\end{array}$} & Q25 & 2.8 & 3.4 & 3.7 & 3.5 & 2.8 & 2.9 & 3.3 & 2.8 & 4.6 \\
\hline & & $\mu$ & 3.1 & 3.9 & 4.1 & 4.2 & 3.1 & 3.2 & 3.6 & 3.0 & 4.9 \\
\hline & & Q75 & 3.5 & 4.3 & 4.6 & 4.8 & 3.5 & 3.4 & 3.9 & 3.3 & 5.3 \\
\hline & & KW & $\mathrm{a}$ & bd & b & $\mathrm{b}$ & $\mathrm{ac}$ & $\mathrm{a}$ & $\mathrm{d}$ & $\mathrm{ac}$ & $\mathrm{e}$ \\
\hline & \multirow{4}{*}{$\begin{array}{c}\text { Vessel-ray pits }(\mu \mathrm{m}) \\
(\mathbf{N} 125)\end{array}$} & Q25 & 2.9 & 3.3 & 3.8 & 3.6 & 2.9 & 2.8 & 2.9 & 2.8 & 4.5 \\
\hline & & $\mu$ & 3.1 & 3.8 & 4.2 & 4.1 & 3.2 & 3.1 & 3.3 & 3.0 & 4.8 \\
\hline & & Q75 & 3.5 & 4.3 & 4.6 & 4.7 & 3.5 & 3.5 & 3.8 & 3.3 & 5.2 \\
\hline & & KW & ad & b & c & $\mathrm{bc}$ & $\mathrm{ad}$ & ad & a & $\mathrm{d}$ & e \\
\hline \multirow{20}{*}{$\stackrel{\overrightarrow{0}}{\text { 它 }}$} & \multirow{4}{*}{$\begin{array}{c}\text { Diameter }(\mu \mathrm{m}) \\
(\mathrm{N} \mathrm{125})\end{array}$} & Q25 & 22.8 & 29.4 & 30.9 & 19.6 & 21.3 & 19.6 & 22.8 & 24.4 & 28.7 \\
\hline & & $\mu$ & 23.4 & 32.0 & 34.8 & 22.1 & 22.2 & 21.3 & 27.2 & 26.9 & 33.6 \\
\hline & & Q75 & 31.1 & 37.3 & 37.1 & 25.2 & 28.2 & 22.4 & 29.5 & 28.6 & 38.4 \\
\hline & & KW & $\mathrm{a}$ & $\mathrm{d}$ & $\mathrm{d}$ & b & c & b & $\mathrm{ac}$ & $\mathrm{ac}$ & $\mathrm{d}$ \\
\hline & \multirow{4}{*}{$\begin{array}{l}\text { Lumen diam. }(\mu \mathrm{m}) \\
\qquad(\mathrm{N} \mathrm{125})\end{array}$} & Q25 & 14.7 & 16.8 & 17.8 & 13.8 & 12.9 & 11.2 & 16.4 & 14.4 & 19.6 \\
\hline & & $\mu$ & 19.8 & 18.9 & 19.4 & 15.1 & 15.6 & 12.9 & 18.8 & 16.3 & 24.8 \\
\hline & & Q75 & 23.1 & 25.1 & 22.3 & 17.5 & 19.4 & 14.3 & 21.4 & 17.7 & 30.5 \\
\hline & & KW & $\mathrm{a}$ & $\mathrm{a}$ & $\mathrm{ac}$ & b & b & $\mathrm{d}$ & a & b & c \\
\hline & \multirow{4}{*}{$\begin{array}{l}\text { Length }(\mu \mathrm{m}) \\
\quad(\mathbf{N} 125)\end{array}$} & Q25 & 1388 & 1420 & 1459 & 1115 & 1046 & 1002 & 1278 & 1330 & 1199 \\
\hline & & $\mu$ & 1506 & 1543 & 1553 & 1249 & 1170 & 1063 & 1363 & 1411 & 1271 \\
\hline & & Q75 & 1647 & 1614 & 1711 & 1342 & 1289 & 1150 & 1481 & 1514 & 1388 \\
\hline & & KW & $\mathrm{a}$ & $\mathrm{a}$ & $\mathrm{a}$ & b & b & $\mathrm{d}$ & c & c & $\mathrm{b}$ \\
\hline & \multirow{4}{*}{$\begin{array}{l}\text { Wall thickness }(\mu \mathrm{m}) \\
\text { (N 125) }\end{array}$} & Q25 & 3.77 & 5.68 & 6.32 & 2.88 & 4.09 & 3.58 & 3.39 & 4.81 & 3.54 \\
\hline & & $\mu$ & 4.2 & 6.4 & 7.3 & 3.5 & 4.5 & 4.1 & 3.8 & 5.3 & 4.0 \\
\hline & & Q75 & 4.6 & 7.2 & 7.9 & 4.2 & 5.0 & 4.8 & 4.2 & 5.6 & 4.4 \\
\hline & & KW & $\mathrm{a}$ & c & c & $\mathrm{b}$ & $\mathrm{a}$ & $\mathrm{a}$ & $a b$ & $\mathrm{~d}$ & $\mathrm{ab}$ \\
\hline & & Q25 & 3.20 & 4.36 & 3.68 & 2.71 & 3.48 & 2.43 & 2.85 & 2.14 & 6.58 \\
\hline & Pits $(\mu \mathrm{m})$ & $\mu$ & 3.56 & 4.88 & 4.19 & 3.34 & 4.02 & 2.79 & 3.36 & 2.38 & 7.17 \\
\hline & (N 125) & Q75 & 3.9 & 5.9 & 4.7 & 3.9 & 4.7 & 3.2 & 3.9 & 2.9 & 8.3 \\
\hline & & KW & $\mathrm{a}$ & $\mathrm{d}$ & b & $\mathrm{a}$ & b & c & $\mathrm{a}$ & c & $\mathrm{e}$ \\
\hline & & Q25 & 8 & 4 & 2 & 3 & 3 & 5 & 5 & 3 & 4 \\
\hline & Rays/mm' & $\mu$ & 8 & 5 & 3 & 4 & 3 & 5 & 5 & 4 & 4 \\
\hline & (N 100) & Q75 & 9 & 5 & 3 & 4 & 4 & 6 & 6 & 4 & 5 \\
\hline & & KW & $\mathrm{a}$ & $\mathrm{d}$ & c & $\mathrm{b}$ & $\mathrm{bc}$ & $\mathrm{d}$ & $\mathrm{d}$ & $\mathrm{bc}$ & $\mathrm{e}$ \\
\hline & & Q25 & 475 & 924 & 768 & 516 & 466 & 411 & 475 & 406 & 464 \\
\hline 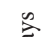 & Height $(\mu \mathrm{m})$ & $\mu$ & 597 & 1187 & 987 & 584 & 553 & 510 & 548 & 503 & 689 \\
\hline$\widetilde{\simeq}$ & $(\mathbf{N} 125)$ & Q75 & 750 & 1351 & 1102 & 742 & 685 & 633 & 747 & 668 & 823 \\
\hline & & KW & $\mathrm{a}$ & $\mathrm{b}$ & $\mathrm{b}$ & $\mathrm{a}$ & $\mathrm{a}$ & $\mathrm{a}$ & $\mathrm{a}$ & $\mathrm{a}$ & $\mathrm{a}$ \\
\hline & & Q25 & 23.2 & 37.3 & 65.6 & 37.4 & 23.1 & 23.2 & 33.8 & 24.0 & 58.7 \\
\hline & Width $(\mu \mathrm{m})$ & $\mu$ & 28.4 & 44.5 & 69.5 & 42.8 & 26.7 & 26.7 & 39.2 & 26.7 & 69.3 \\
\hline & (N 125) & Q75 & 34.0 & 52.8 & 76.5 & 50.1 & 30.2 & 30.3 & 48.9 & 29.3 & 78.2 \\
\hline & & KW & $\mathrm{a}$ & $\mathrm{b}$ & c & $\mathrm{b}$ & $\mathrm{a}$ & $\mathrm{a}$ & $\mathrm{b}$ & $\mathrm{a}$ & c \\
\hline
\end{tabular}



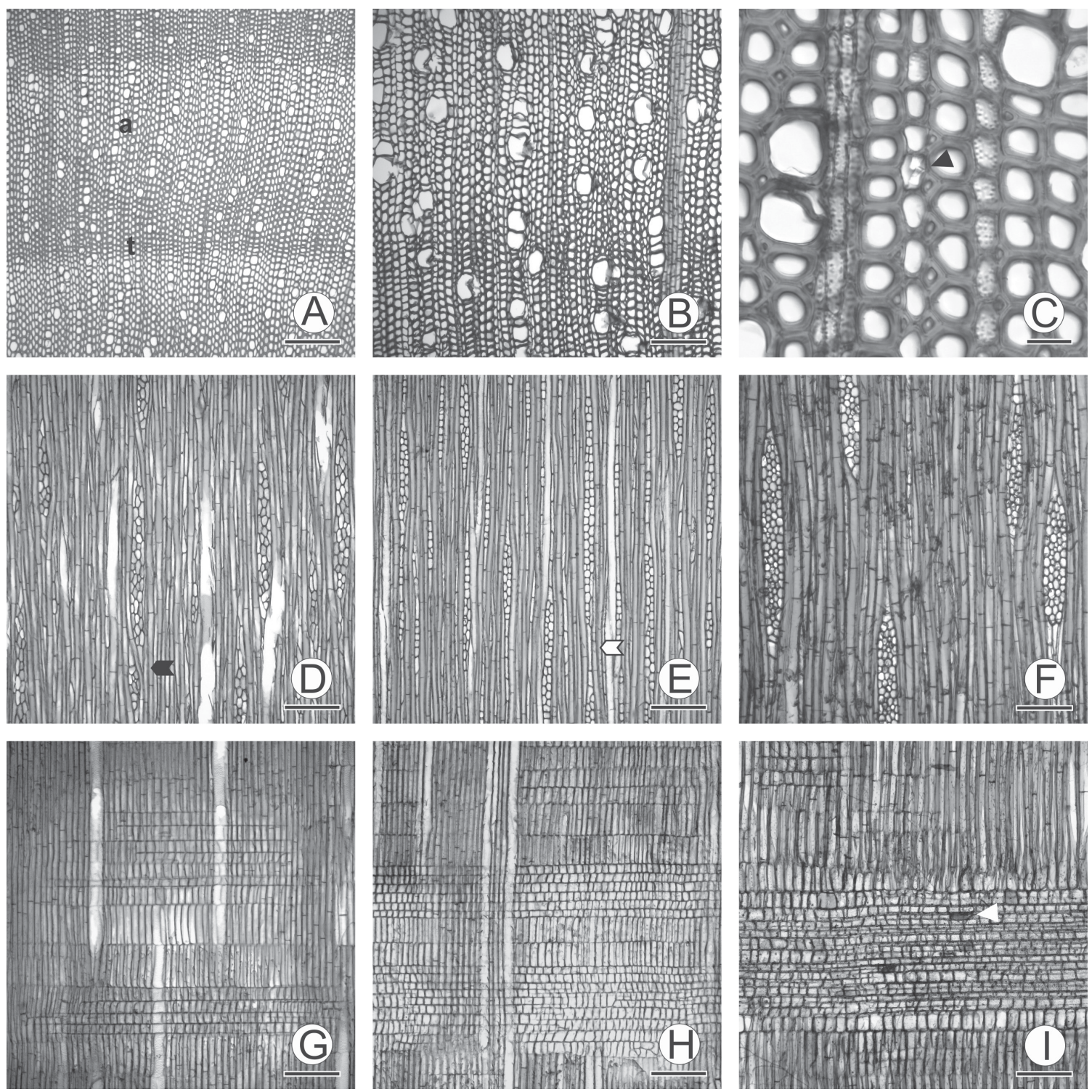

Figure 1. Light microscopy micrographs of Psychotria woods sections. A: P. deflexa, distinct growth rings, diffuse porosity and vessel arrangement in a radial pattern $(\mathrm{e}=$ initial wood, $\mathrm{l}=$ later wood). B: $P$. vellosiana, diffuse porosity and vessel arrangement in a radial pattern. C: $P$. leiocarpa, vessels with circular to oval outline, libriform fibers thin- to thick-walled and prismatic crystals inside ray cells (arrowhead). D: $P$. nuda, multiseriate and aggregate rays with uniseriate portions (arrowhead). E: P. hoffmannseggiana, uniseriate and biseriate rays, and fused rays (arrowhead). F: P. vellosiana, multiseriate rays with sheath cells and septate fiber-tracheids (arrow). G: P. ruelliifolia, rays composed of procumbent, square and upright cells. H: P. hoffmannseggiana, rays composed of square and/or upright cells. I: P. carthagenensis, rays composed of procumbent, square and upright cells, and raphides crystals inside procumbent ray cells (arrowhead). Scale bar: A, B and $\mathrm{D}$ to $\mathrm{I}=100 \mu \mathrm{m} ; \mathrm{C}=20 \mu \mathrm{m}$.

\section{Descriptions of studied species}

Psychotria deflexa DC. (Figs. 1A, 2C, 3D)

Growth Rings: distinct or slightly distinct, marked by thick-walled and radially flattened latewood versus thin-walled earlywood fibers.

Vessels: diffuse-porous, 140 vessels $/ \mathrm{mm}^{2}$; solitary, radial multiple of 2-6 or in cluster of 3-4 elements; circular to oval outline; mean length $829.56 \mu \mathrm{m}$; mean tangential diameter $25.36 \mu \mathrm{m}$; mean wall thickness $3.05 \mu \mathrm{m}$; simple perforation plates and 1-2 lateral perforation plates; appendices on both ends; minute $(<4 \mu \mathrm{m})$, alternate, circular and vestured intervessel pits with distinct borders; vessel-ray pits similar to intervessel pits; tyloses and gums present.

Fibers: septate, mean length $1187.65 \mu \mathrm{m}$; mean diameter $22.25 \mu \mathrm{m}$; lumina $16.14 \mu \mathrm{m}$; thin- to thick-walled; mean size of pit $4.02 \mu \mathrm{m}$.

Axial Parenchyma: extremely rare.

Rays: average $3 / \mathrm{mm}$; uniseriate and biseriate with uniseriate portions, composed of square and/or upright cells; mean width $26.84 \mu \mathrm{m}$, mean length $583.75 \mu \mathrm{m}$; fused rays and disjunctive cell wall present. One or more prismatic crystals per cell are present. 

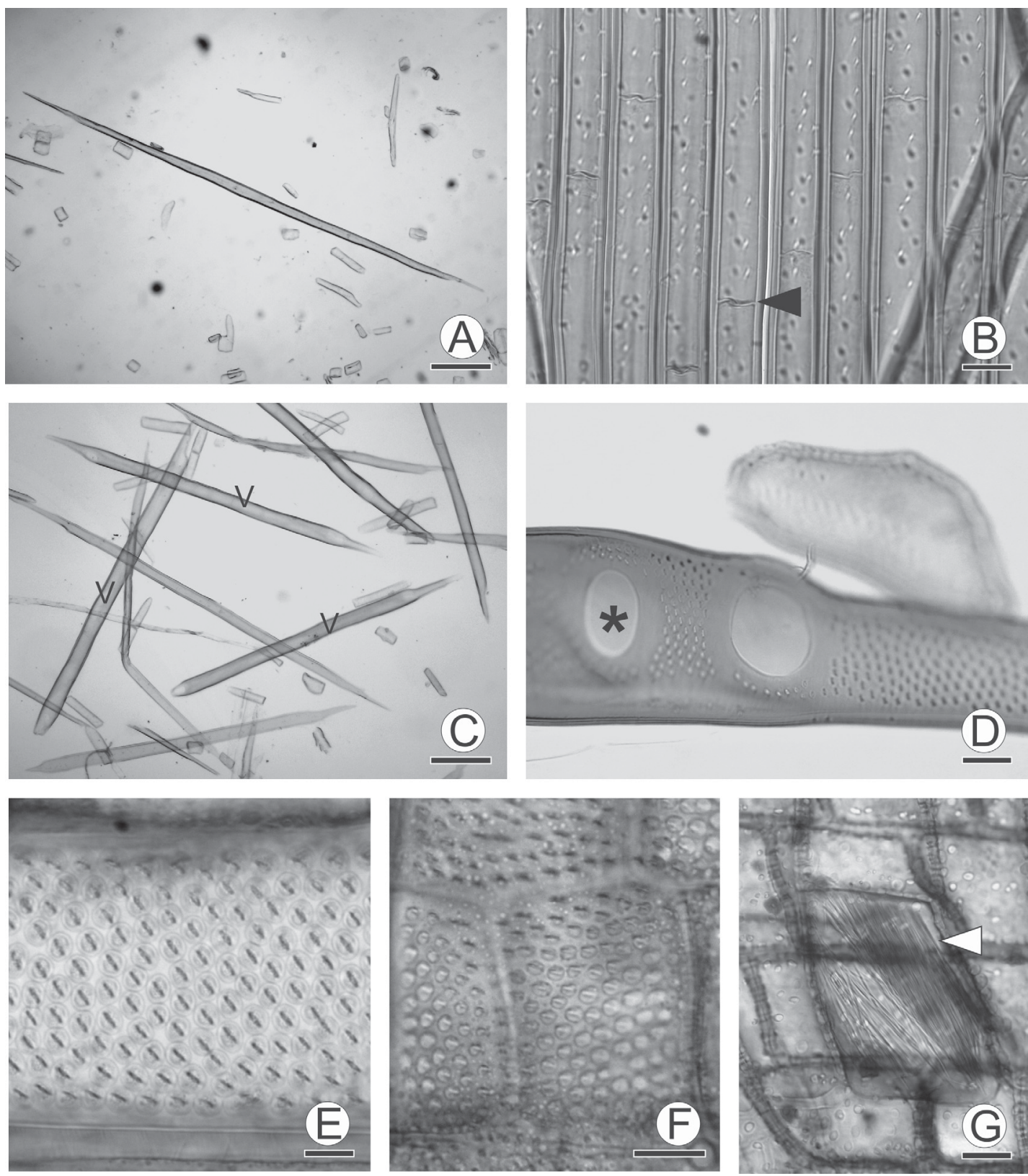

Figure 2. Light microscopy micrographs. A: P. brasiliensis, fiber-tracheids. B: P. hoffmannseggiana, septate fiber-tracheids (arrowhead), simple fiber pits and fiberwall thickness. C: P. deflexa, vessel elements (V). D: P. vellosiana, appendices and simple perforation plates on terminal and lateral wall of vessel $\left({ }^{*}\right)$. E: P. carthagenensis, intervessel vestured pits. F: P. vellosiana, ray-vessel vestured pits. G: P. carthagenensis, raphides crystals inside the upright ray cell (arrowhead). Scale bar: $\mathrm{A}$ and $\mathrm{C}=100 \mu \mathrm{m} ; \mathrm{B}, \mathrm{D}, \mathrm{E}, \mathrm{F}$ and $\mathrm{G}=20 \mu \mathrm{m}$.

Psychotria vellosiana Benth. (Figs. 1B, 1F, 2D, 2F)

Growth Rings: distinct or slightly distinct, marked by thick-walled and radially flattened latewood versus thin-walled earlywood fibers.

Vessels: diffuse-porous, 28 vessels $/ \mathrm{mm}^{2}$; solitary, radial multiple of 2-5 or in cluster of 3-4 elements; circular to oval outline; mean length $860.11 \mu \mathrm{m}$; mean tangential diameter $68.58 \mu \mathrm{m}$; mean wall thickness $1.81 \mu \mathrm{m}$; simple perforation plates and 1-2 lateral perforation plates; appendices on both ends; small (4-7 $\mu \mathrm{m})$, alternate, circular and vestured intervessel pits with distinct borders; vessel-ray pits similar to intervessel pits.

Fibers: septate, mean length $1281.81 \mu \mathrm{m}$; mean diameter $32.92 \mu \mathrm{m}$; lumina $24.92 \mu \mathrm{m}$; thin- to thick-walled; mean size of pit $7.17 \mu \mathrm{m}$.

Axial Parenchyma: extremely rare.

Rays: average 4/mm; multiseriate (3-6 cells wide), with uniseriate portions, composed of procumbent, square and upright cells; mean wide $68.54 \mu \mathrm{m}$; mean length $665.65 \mu \mathrm{m}$; sheath cells and disjunctive cell wall present, fused and aggregate rays present. 

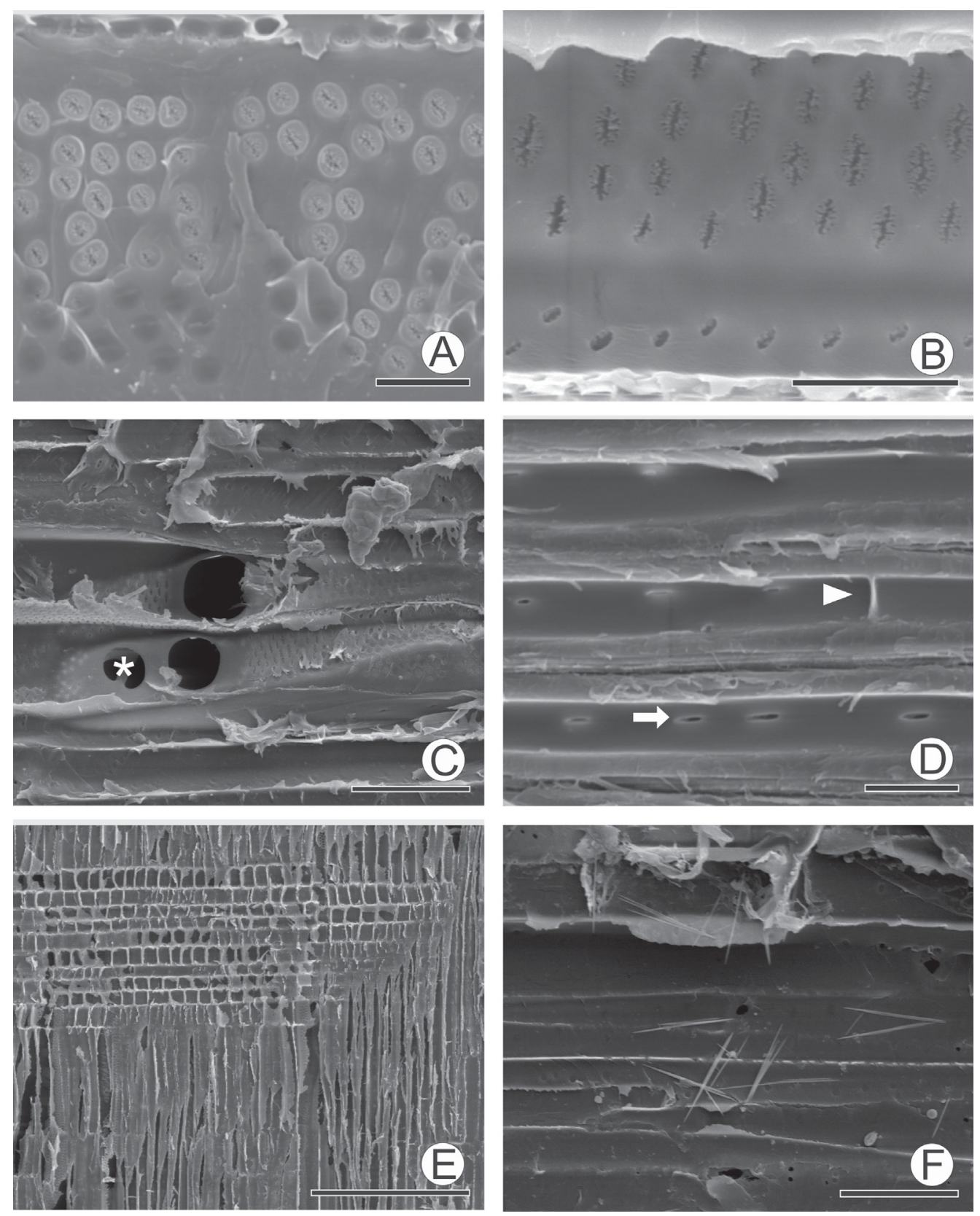

Figure 3. Scanning electron microscopy micrographs. A: P. brasiliensis, vestured intervessel pits viewed from outside the vessel. B: P. pubigera, vestured intervessel pits viewed from inside vessel. C: P. ruelliifolia, vessel with a simple perforation plate on terminal and lateral wall $\left({ }^{*}\right)$ and vessel appendices. D: $P$. deflexa, septate fiber-tracheids (arrowhead) with simple fiber pits (arrow). E: P. pubigera, ray composition of only square and upright cells. F: $P$. ruelliifolia, raphides crystals coming out from upright ray cells (arrowhead). Scale bar: $A$ and $B=10 \mu \mathrm{m} ; C, D$ and $F=50 \mu \mathrm{m} ; \mathrm{E}=200 \mu \mathrm{m}$.

Psychotria leiocarpa Cham. \& Schlecht. (Fig. 1C)

Growth Rings: distinct or slightly distinct, marked by thick-walled and radially flattened latewood versus thin-walled earlywood fibers.

Vessels: diffuse-porous, 178 vessels $/ \mathrm{mm}^{2}$; solitary, radial multiple of 2-5 or in cluster of 3-4 elements (rare); circular to oval outline; mean length $716.93 \mu \mathrm{m}$; mean tangential diameter $23.75 \mu \mathrm{m}$; mean wall thickness 3.12 $\mu \mathrm{m}$; simple perforation plates and 1-2 lateral perforation plates; appendices on both ends; minute $(<4 \mu \mathrm{m})$, alternate, circular and vestured intervessel pits with distinct borders; vessel-ray pits similar to intervessel pits; tyloses present.

Fibers: septate, mean length $1076.12 \mu \mathrm{m}$; mean diameter $21.04 \mu \mathrm{m}$; lumina $12.75 \mu \mathrm{m}$; thin- to thick-walled; mean size of pit $2.79 \mu \mathrm{m}$.

Axial Parenchyma: extremely rare.

Rays: average $5 / \mathrm{mm}$; multiseriate (2-4 cells wide) with uniseriate portions, composed of square and/or upright cells; mean width $27.27 \mu \mathrm{m}$ mean length $552.73 \mu \mathrm{m}$; ag- 
gregate and fused rays present. One or more prismatic crystals per cell and raphides crystals are present in upright cells.

Psychotria nuda Cham. \& Schlecht. (Fig. 1D)

Growth Rings: distinct or slightly distinct, marked by thick-walled and radially flattened latewood versus thin-walled earlywood fibers.

Vessels: diffuse-porous, 173 vessels $/ \mathrm{mm}^{2}$; solitary, radial multiple of 2-6 or in cluster of 3-5 elements; circular to oval outline; mean length $892.18 \mu \mathrm{m}$; mean tangential diameter $27.03 \mu \mathrm{m}$; mean wall thickness $2.18 \mu \mathrm{m}$; simple perforation plates and 1-2 lateral perforation plates; appendices on both ends; minute $(<4 \mu \mathrm{m})$, alternate, circular and vestured intervessel pits with distinct borders; vessel-ray pits similar to intervessel pits.

Fibers: septate, mean length $1376.74 \mu \mathrm{m}$; mean diameter $25.63 \mu \mathrm{m}$; lumina $19.39 \mu \mathrm{m}$; thin- to thick-walled; mean size of pit $3.36 \mu \mathrm{m}$.

Axial Parenchyma: extremely rare.

Rays: average $5 / \mathrm{mm}$; multiseriate (2-4 cells wide) with uniseriate portions, composed of procumbent, square and upright cells; mean width $40.70 \mu \mathrm{m}$; mean length $616.38 \mu \mathrm{m}$; aggregate and fused rays present.

Psychotria hoffmannseggiana Müll. Arg. (Figs. 1E, 1H, 2B) Growth Rings: distinct or slightly distinct, marked by thick-walled and radially flattened latewood versus thin-walled earlywood fibers.

Vessels: diffuse-porous; 183 vessels $/ \mathrm{mm}^{2}$; solitary, radial multiple of 2-5 or in cluster of 3-4 elements; circular to oval outline; mean length $1102.94 \mu \mathrm{m}$; mean tangential diameter $28.37 \mu \mathrm{m}$; mean wall thickness 2.87 $\mu \mathrm{m}$; simple perforation plates and 1-2 lateral perforation plates; appendices on both ends; minute $(<4 \mu \mathrm{m})$, alternate, circular and vestured intervessel pits with distinct borders; vessel-ray pits similar to intervessel pits; tyloses present.

Fibers: septate, mean length $1506.64 \mu \mathrm{m}$; mean diameter $22.57 \mu \mathrm{m}$; lumina $19.88 \mu \mathrm{m}$; thin- to thick-walled; mean size of pit $3.56 \mu \mathrm{m}$.

Axial Parenchyma: extremely rare.

Rays: average $8 / \mathrm{mm}$; uniseriate and biseriate with uniseriate portions, composed of square and/or upright cells; mean width $28.55 \mu \mathrm{m}$; mean length $617.79 \mu \mathrm{m}$; fused rays present.

Psychotria ruelliifolia Cham. \& Schltdl. (Figs. 1G, 3C, 3F) Growth Rings: Indistinct.

Vessels: diffuse-porous, 90 vessels $/ \mathrm{mm}^{2}$; solitary, radial multiple of 2-5 or in cluster of 3-5 elements; angular outline; mean length $898.13 \mu \mathrm{m}$; mean tangential diameter 33.95 $\mu \mathrm{m}$; mean wall thickness $2.87 \mu \mathrm{m}$; simple perforation plates and 1-2 lateral perforation plates; appendices on both ends; small (4-7 $\mu \mathrm{m})$, alternate, circular and vestured intervessel pits with distinct borders; vessel-ray pits similar to intervessel pits.

Fibers: septate, mean length $1243.90 \mu \mathrm{m}$; mean diameter $22.54 \mu \mathrm{m}$; lumina $15.47 \mu \mathrm{m}$; thin- to thick-walled; mean size of pit $3.34 \mu \mathrm{m}$.

Axial Parenchyma: extremely rare.

Rays: average $4 / \mathrm{mm}$; multiseriate ( $2-4$ cells wide) composed of body cells procumbent and square with marginal rows of or upright cells; mean width $44.70 \mu \mathrm{m}$; mean length $629.81 \mu \mathrm{m}$; fused and aggregate rays present. Raphides crystals are present in procumbent and upright cells.

Psychotria carthagenensis Jacq. (Figs. 1I, 2E, 2G)

Growth Rings: Indistinct.

Vessels: diffuse-porous, 26 vessels $/ \mathrm{mm}^{2}$; solitary, radial multiple of 2-5 or in cluster of 3-4 elements; circular to oval outline; mean length $952.48 \mu \mathrm{m}$; mean tangential diameter $54.57 \mu \mathrm{m}$; mean wall thickness $3.64 \mu \mathrm{m}$; simple perforation plates and lateral perforation plates; appendices on both ends; small $(4-7 \mu \mathrm{m})$, alternate, circular and vestured intervessel pits with distinct borders; vessel-ray pits similar to intervessel pits; tyloses and gums present.

Fiber: septate, mean length $1571.12 \mu \mathrm{m}$; mean diameter $35.15 \mu \mathrm{m}$; lumina $20.86 \mu \mathrm{m}$; thin- to thick-walled; mean size of pit $4.19 \mu \mathrm{m}$.

Axial Parenchyma: extremely rare.

Rays: average $8 / \mathrm{mm}$; uniseriate and biseriate with uniseriate portions, composed of square and/or upright cells and multiseriate sections (3-8 cells wide) composed of body cells procumbent and square with marginal rows or upright cells; mean width $73.63 \mu \mathrm{m}$; mean length $617.79 \mu \mathrm{m}$; sheath cells and disjunctive cell wall present, fused and aggregate rays present. Raphides crystals are present in procumbent, square and upright cells.

\section{Psychotria brasiliensis Vell. (Figs. 2A, 3A)}

Growth Rings: distinct or slightly distinct, marked by thick-walled and radially flattened latewood versus thin-walled earlywood fibers.

Vessels: diffuse-porous, 67 vessels $/ \mathrm{mm}^{2}$; solitary, radial multiple of 2-5 or in clusters of 3-4 elements; circular to oval outline; mean length $1152.84 \mu \mathrm{m}$; mean tangential diameter $37.10 \mu \mathrm{m}$; mean wall thickness $2.16 \mu \mathrm{m}$; simple perforation plates and 1-2 lateral perforation plates; appendices on both ends; minute $(<4 \mu \mathrm{m})$, alternate, circular and vestured intervessel pits with distinct borders; vessel-ray pits similar to intervessel pits.

Fibers: septate, mean length $1507.47 \mu \mathrm{m}$; mean diameter $33.18 \mu \mathrm{m}$; lumina $20.38 \mu \mathrm{m}$; thin- to thick-walled; mean size of pit $4.88 \mu \mathrm{m}$.

Axial Parenchyma: extremely rare.

Rays: average $5 / \mathrm{mm}$; multiseriate ( $2-4$ cells wide) with uniseriate portions, composed of body cells procumbent 
with marginal rows of square or upright cells; mean width $44.85 \mu \mathrm{m}$; mean length $1167.24 \mu \mathrm{m}$; disjunctive cell wall and fused rays present.

Psychotria pubigera Schltdl. (Fig. 3B, 3E)

Growth Rings: distinct or slightly distinct, marked by thick-walled and radially flattened latewood versus thin-walled earlywood fibers.

Vessels: diffuse-porous, 105 vessels $/ \mathrm{mm}^{2}$; solitary, radial multiple of 2-5 or in cluster of 3-4 elements (rare); angular outline; mean length $952.68 \mu \mathrm{m}$; mean tangential diameter $32.46 \mu \mathrm{m}$; mean wall thickness 2.44 $\mu \mathrm{m}$; simple perforation plates and 1-2 lateral perforation plates; appendices on both ends; minute $(<4 \mu \mathrm{m})$, alternate, circular and vestured intervessel pits with distinct borders; vessel-ray pits similar to intervessel pits; tyloses present.

Fibers: septate, mean length $1408.77 \mu \mathrm{m}$; mean diameter $26.49 \mu \mathrm{m}$; lumina $16.05 \mu \mathrm{m}$; thin- to thick-walled; mean size of pit $2.38 \mu \mathrm{m}$.

Axial Parenchyma: extremely rare.

Rays: average $4 / \mathrm{mm}$; exclusively biseriate, composed of square and upright cells; mean width $26.60 \mu \mathrm{m}$; mean length $556.70 \mu \mathrm{m}$; fused rays present.

\section{Qualitative Comparative Analysis}

Cluster analysis (Fig. 4) separated Coussarea friburguensis from the remaining species, which separated into three groups with more than $40 \%$ similarity. The group with greatest similarity was composed of $P$. hoffmannseggiana,
P. pubigera, P. deflexa and P. leiocarpa. A second group was contained $P$. brasiliensis, $P$. nuda and $P$. ruelliifolia, and a third group contained $P$. carthagenensis, $P$. vellosiana and Coussarea congestiflora.

The principal component analysis (Fig. 5) separated Coussarea friburguensis from the remaining species, and the closest group was again $P$. hoffmannseggiana, P. pubigera, $P$. deflexa and P. leiocarpa. The other species did not form a cohesive group despite $P$. vellosiana and $C$. congestiflora being closer than in the cluster analysis. The first two axes of the principal component analysis of the qualitative data explained $47.35 \%$ of the total variance among species. Axis 1 accounted for $27.44 \%$ of the total variance and separated species mainly according to scalariform or simple perforation plates, angular or circular vessel outline, intervessel pit size, ray width, septate fiber presence, fiber length and growth ring distinctiveness. Axis 2 explained $19.91 \%$ of the total variance and separated species mainly by width of the fiber wall and the fiber pits, presence of sheath cells in rays and cellular composition of rays.

\section{Quantitative Comparative Analysis}

The first two axes of the principal component analysis of the quantitative data explained $55.29 \%$ of the total variance among species (Fig. 6). Axis 1 accounted for $40.37 \%$ of the total variance and separated the species mainly by vessel frequency, ray frequency, vessel mean wall thickness and vessel length. Axis 2 explained $14.92 \%$ of the total variance and separated the species mainly by intervessel pit size and vessel mean tangential diameter.

\section{UPGMA}

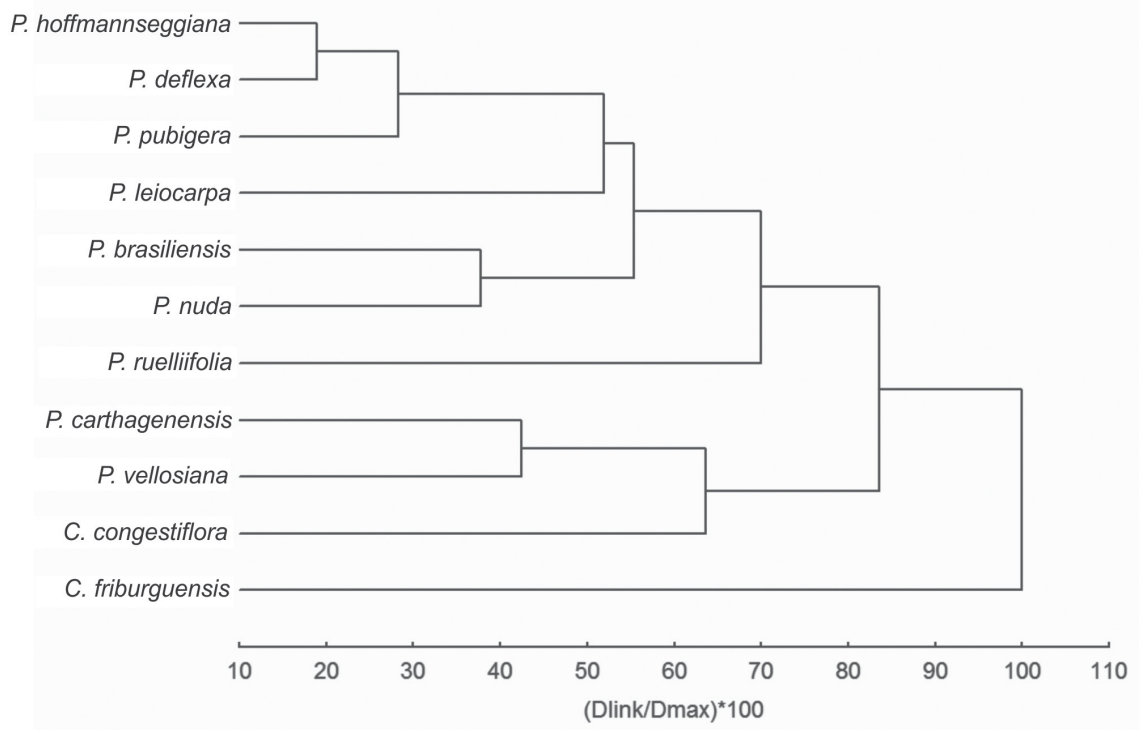

Figure 4. Cluster similarity analysis of Psychotria and Coussarea qualitative characters. 


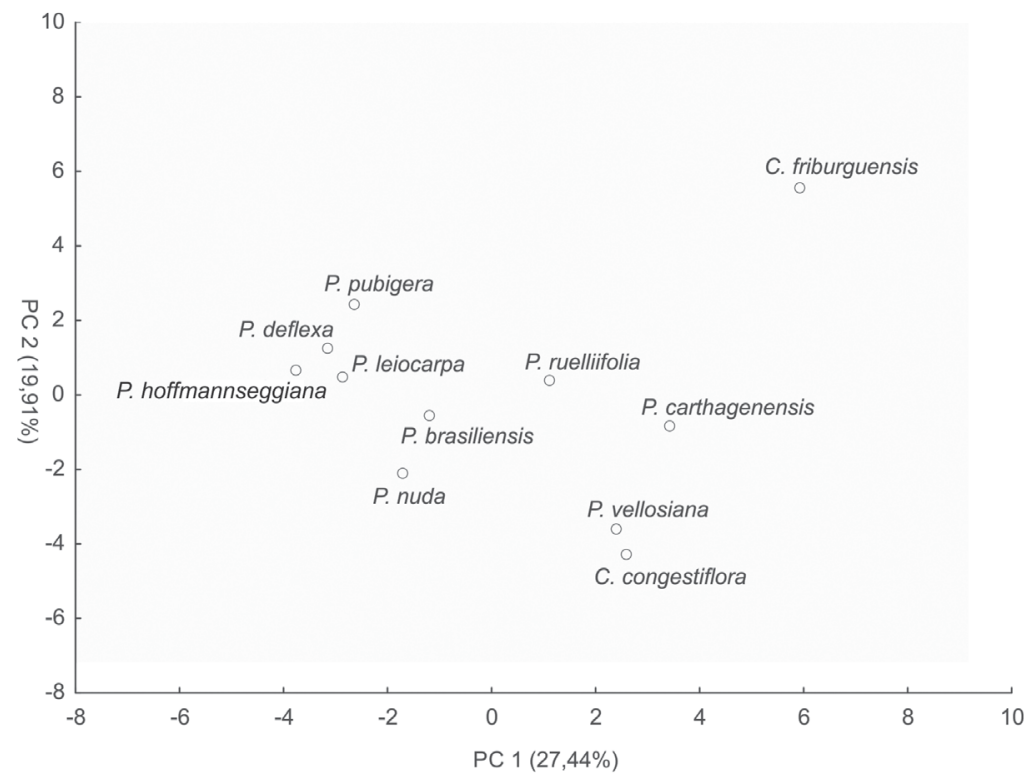

Figure 5. Principal Component Analysis of Psychotria and Coussarea qualitative characters.

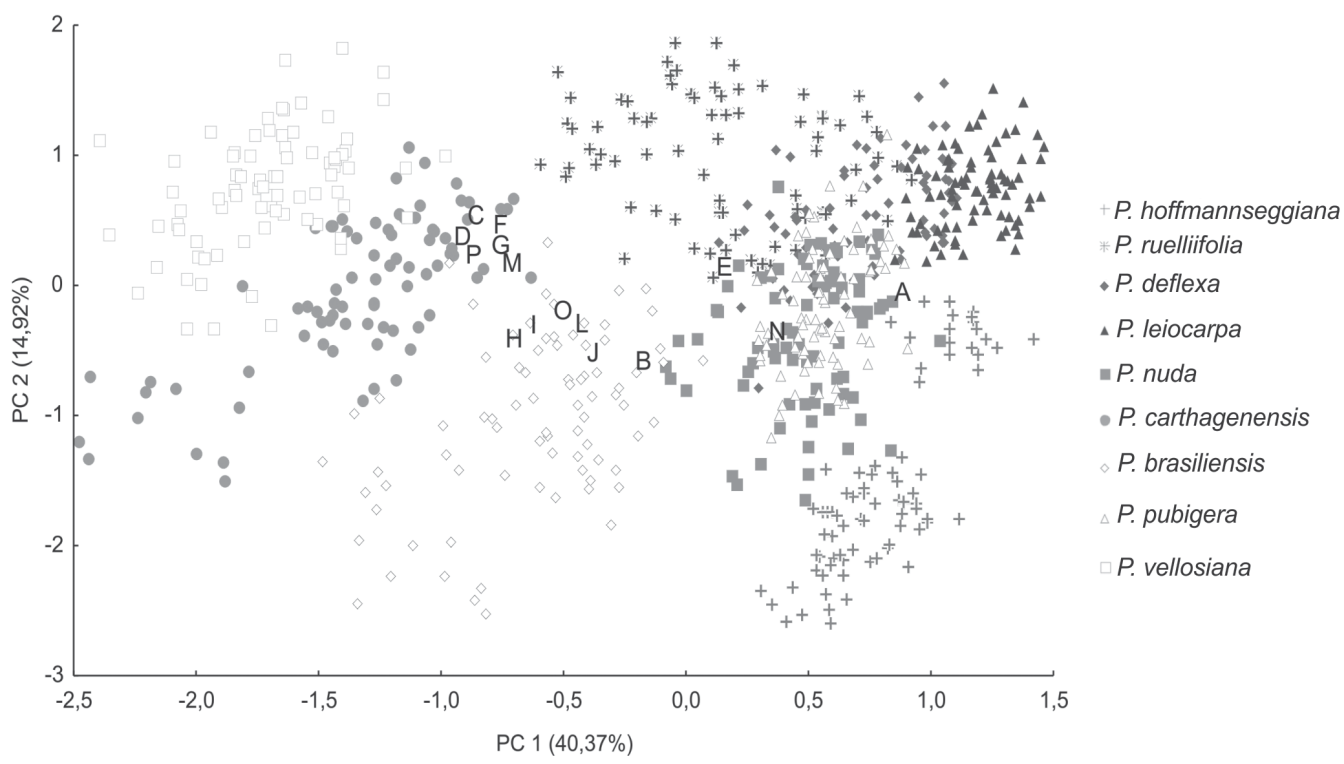

Figure 6. Principal Component Analysis of Psychotria quantitative characters. $\mathrm{A}=$ Vessels $/ \mathrm{mm}^{2}, \mathrm{~B}=$ vessel length, $\mathrm{C}=$ tangential diameter of vessel, $\mathrm{D}=$ radial diameter of vessel, $\mathrm{E}=$ vessel wall thickness, $\mathrm{F}=$ intervessel pits, $\mathrm{G}=$ vessel-ray pits, $\mathrm{H}=$ fiber diameter, $\mathrm{I}=$ fiber lumina, $\mathrm{J}=$ fiber length, $\mathrm{L}=$ fiber wall thickness, $\mathrm{M}=$ fiber pits, $\mathrm{N}=$ rays $/ \mathrm{mm}, \mathrm{O}=$ ray wide, $\mathrm{P}$ = ray length.

\section{Discussion}

In general, the anatomical characters observed in the present study are classified as type II (Koek-Noorman 1977), corroborating the results of Jansen et al. (2002) who defended the placement of the genus Psychotria in the tribe Psychotrieae and the subfamily Rubioideae. The type II characters observed in the studied species were: vessel elements solitary, in radial multiples and clusters of a few elements; libriform and septate fibers; axial parenchyma absent or extremely rare; simple perforation plates; vestured vessel-ray parenchyma and intervessel-ray pits.

Although the species of Psychotria mainly occur in humid areas of the understory, the presence of narrow and numerous vessel elements is a characteristic of the genus and indicative of adaptation to conditions of water deficit with a preference for conductive security instead of efficiency.

The presence or absence of different types of mineral inclusions in the ray cells is an important parameter for identifying wood and serves as a taxonomic and systematic 
marker for Rubiaceae anatomy (Jansen et al. 2002; Callado \& Silva Neto 2003). Raphide crystals are commonly found in the wood of the species of Psychotria and they are typical of Rubioideae (Jansen et al. 2001). Raphide crystals were found in the radial cells of $P$. carthagenensis, $P$. ruelliifolia and $P$. leiocarpa. In $P$. deflexed and $P$. leiocarpa one or more prismatic crystals in large ray cells were found. The ecological role of inorganic inclusions in wood is not very clear, but the process of formation and the shape of crystals are directly influenced by genetic factors (Luchi 1990; Nakata 2012), which strengthens their taxonomic significance.

Vestured intervessel pits are important features of $\mathrm{Ru}$ biaceae and their structure and size are of great diagnostic value for the family (Jansen et al. 2001). The presence of vestured pits is important in making a plant less vulnerable to embolism and consequent disruption in water transport (Tyree \& Sperry 1989; Dickison 2000). However, more important than the size is the complex structure of the membrane pit (Sano 2004). According to Williams \& Leon (2005), the size of pits and their structure are more related to the taxonomic group than to environmental conditions. All the species studied here exhibited vestured pits, but no correlation was observed regarding their size or structure.

The presence of septate fibers in the studied species may also be related to the high frequency and small-diameter of vessels and the extremely rare axial parenchyma. Substance transport and water conduction explain the presence of fibers with large lumen, thin walls and relatively large pits among the studied species (Jansen et al. 2002).

The habit of an individual plant can exert significant influence on the anatomical features of their wood. According to Lens et al. (2004), plants of shrubby habit have some anatomical features similar to those of plants of arboreous habit that are adapted to water deficit. Nevertheless, such features should not be interpreted as having adaptive value to xeromorphy, but instead to the habit of the species. When compared to shrubs, trees generally have larger, longer and less frequent vessels (Lens et al. 2004). In this work, the diameter and frequency of the vessels can also be related to the habit of the species, and among the nine species studied, $P$. carthagenensis and $P$. vellosiana are trees, while the remainder are small treelets or shrubs.

The width of rays may also be related to habit. According to Jansen et al. (2002), the presence of biseriate and multiseriate rays 2-4 cells wide and uniseriate portions at the margins is common among species of Psychotria, whereas species with exclusively uniseriate rays and multiseriate rays of more than four cells wide, as observed in P. carthagenensis and $P$. vellosiana, is less common. Since arboreous habit is observed only in these two species, it is possible that the structure of the rays can also be influenced by size of the species.

The results of the principal component analyses of qualitative and quantitative characters were similar, both separating $P$. carthagenensis and $P$. vellosiana from the remaining species. This separation is mainly due to these two species having less frequent and larger diameter vessels, and consequently greater vulnerability and mesomorphic indices. It is possible that these attributes are related to the maximum height of these two species compared to that of the others. $P$. nuda, $P$. hoffmannseggiana, $P$. pubigera, $P$. deflexa and $P$. leiocarpa were grouped together mainly because they have small diameters and high-frequency vessels.

The nine species of Psychotria were segregated by wood anatomy. Although, the characters of vessel frequency, length and diameter, and vulnerability and mesomorphic indices can be susceptible to environmental changes, and most likely have a direct relationship with growth habit (Carlquist 2001; Sperry 2003; Lens et al. 2004), they were useful as diagnostic characters in the present study since all samples were obtained from the same study site. However, the use of these characters as diagnostic must be done with caution and should not be used in phylogenetic analyses of the group.

The use of the two species of Coussarea, belonging to Rubioideae, as outgroups for cluster analysis allowed a clearer recognition of the differences between the species of Psychotria because Coussarea is phylogenetically close to Psychotria. The cluster analysis separated Coussarea friburguensis from the other species, and the remaining species were separated into three groups with more than $40 \%$ similarity. Cluster analysis showed certain differences in the proximity of species of Psychotria from the principal component analysis, especially with regards to the proximity of P. nuda, P. brasiliensis and P. vellosiana and the proximity of $P$. ruelliifolia and P. carthagenensis. These differences between the two analyses may be related to other characteristics, such as the composition of the rays, and the size of fiber pits.

Although P. hoffmannseggiana, P. pubigera, P. deflexa and $P$. leiocarpa are of shrubby habit, they remained as a welldefined group after cluster and grouping analyses without the features that are directly influenced by the habit, such as vessel frequency, length and diameter.

Although Psychotria brasiliensis is a synonym of $P$. nuda (Taylor 2007), we chose to maintain the separated species according to differences observed in wood structure, especially the vessel frequency and length, ray composition and length and presence of disjunctive ray parenchyma in the cell wall present only in P. brasiliensis. The fact that these two species are sympatric, and were sampled under similar environmental conditions, strongly suggests two distinct groups of individuals. Based on this finding, and also on other morphological differences (SJS Neto unpubl.res.), work is being done to revalidate the binomial $P$. brasiliensis.

\section{Conclusion}

Despite the occurrence of many common structural characteristics, the nine species of Psychotria can be segregated by wood anatomy. The diagnostic parameters are the presence or absence of crystals; crystal types (prismatic or 
raphide); cellular composition of rays; presence or absence of disjunctive ray parenchyma in the cell wall; and size and format of vestured pits.

Wood anatomical characters, such as vessel frequency, length and diameter, can be susceptible to environmental influence. In addition, these characters have a direct relationship with the different habits of plants. Thus, the use of these as diagnostic characters must be done with caution even when separate species are being analyzed.

The comparative analyses of both qualitative and quantitative wood anatomical characteristics of the nine species of Psychotria and the two Coussarea outgroups were successful in segregating and grouping the species.

It should be noted that the conclusion that the wood characteristics are more related to the specificities of each studied taxon than to environmental variation is likely due to the sampling of only one study site (Ilha Grande) in the present work.

Nonetheless, wood anatomy proved to be effective in segregating the species of Psychotria, however, phenotypic variation resulting from environmental response deserves greater study because the extent to which these characteristics can vary in each species remains unknown.

\section{Acknowledgements}

The authors are indebted to Conselho Nacional de Desenvolvimento Cientıfico e Tecnológico (CNPq), Fundação de Amparo à Pesquisa do Rio de Janeiro (FAPERJ), Coordenação de Aperfeiçoamento de Pessoal de Nível Superior (CAPES) for their financial support. We thank to Instituto Estadual do Ambiente (INEA) to the plant collecting license and Centro de Estudos Ambientais e Desenvolvimento Sustentável (CEADS), da Universidade do Estado do Rio de Janeiro by field infrastructure. This study is a part of the MSc degree thesis of J.B.C.M. carried out at the Universidade Estadual do Norte Fluminense

\section{References}

Araújo DSD, Oliveira RR. 1988. Reserva Biológica Estadual da Praia do Sul (Ilha Grande, Estado do Rio de Janeiro): lista preliminar da flora. Acta Botanica Brasilica 1: 83-94.

Barros CF, Callado CH, Marcon ML, et al. 2001. Madeiras da Mata Atlântica: Anatomia do lenho de espécies ocorrentes nos remanescentes florestais do estado do Rio de Janeiro - Brasil. Vol. II. Rio de Janeiro, Instituto de Pesquisas Jardim Botânico do Rio de Janeiro.

Bremer B, Eriksson T. 2009. Time tree of Rubiaceae: phylogeny and dating the family, subfamilies, and tribes. International Journal of Plant Science 170: 766-793.

Burger LM, Richter HG. 1991. Anatomia de madeira. São Paulo, Nobel.

Callado CH, Barros AAM, Ribas LA, Albarello N, Gagliardi RF, Jascone CES. 2009. Flora e vegetação da Ilha Grande. In: Bastos MP, Callado CH. (eds.) O ambiente da Ilha Grande. Rio de Janeiro, Universidade do Estado do Rio de Janeiro. p. 95-146.

Callado CH, Silva Neto SJ. 2003. Anatomia do lenho de três espécies do gênero Simira Aubl. (Rubiaceae) da Floresta Atlântica no Estado do Rio de Janeiro. Rodriguésia 54: 23-53.
Carlquist S. 2001. Comparative wood anatomy: Systematic, ecological and evolutionary aspects of Dicotyledon wood. 2nd. edn. Berlín \& Heidelberg, Springer-Verlag.

Davis AP, Bridson D, Jarvis C, Govaerts RL. 2001. The typification and characterization of the genus Psychotria L. (Rubiaceae). Botanical Journal of the Linnean Society 135: 35-42.

Dickison WC. 2000. Integrative plant anatomy. San Diego, Harcourt Academic Press.

Hammer O, Harper DAT, Ryan PD. 2001. PAST: Paleontological Statistics Software Package for Education and Data Analysis. Palaeontologia Electronica 4: 9 .

IAWA Commitee. 1989. List of Microscopic Feature of Hardwood Identification. IAWA Bulletin 10: 219-332.

Jansen S, Robbrecht E, Beeckman H, Smets E. 1997. Wood anatomy of the predominantly African representatives of the tribe Psychotrieae (Rubiaceae-Rubioideae). IAWA Journal 18:169-196.

Jansen S, Baas P, Smets E. 2001. Vestured pits : their occurrence and systematic importance in eudicots. Taxon 50: 135-167.

Jansen S, Robbrecht E, Beeckman H, Smets E. 2002. A survey of the systematic wood of the Rubiaceae. IAWA Journal 23:1-67.

Jansen S, Pletsers A, Sano Y. 2008. The effect of preparation techniques on SEM-imaging of pit membranes. IAWA Journal. 29: 161-178.

Johansen D. 1940. Plant microtechnique. New York, McGraw-Hill Book Company, Inc.

Koek-Noorman J. 1969a. A contribution to the wood anatomy of South American (Chiefly Suriname) Rubiaceae. I. Acta Botanica Neerlandica 18: 108-123.

Koek-Noorman J. 1969b. A contribution to the wood anatomy of South American (Chiefly Suriname) Rubiaceae. II. Acta Botanica Neerlandica 18: 377-395.

Koek-Noorman J. 1977. Systematische Holzanatomie einiger Rubiaceen. Berichte der Deutschen Botanischen Gesellschaft 90: 183-190.

Koek-Noorman J, Hogeweg P. 1974. The wood anatomy of Vanguerieae, Cincohoneae, Codaminae, and Rondeletieae (Rubiaceae). Acta Botanica Neerlandica 23: 627-653.

Koek-Noorman J, Puff C. 1983. The wood anatomy of Rubiaceae tribe Anthospermeae and Paederieae. Plant Systematic and Evolution 143: 17-25.

Koek-Noorman J, Puff C. 1991. The wood anatomy of Paederia L. (Rubiaceae, Paederieae). In: Puff C. (ed.) The genus Paederia L. (Rubiaceae, Paederieae): A multidisciplinary study. Opera Botanica Belgica 3: 35-40.

Lens F, Luteyn JL, Smets E, Jansen S. 2004. Ecological trends in the wood anatomy of Vaccinioideae (Ericaceae s.l.). Flora 199: 309-319.

List of Species of the Brazilian Flora. 2015. Rio de Janeiro Botanical Garden. $<$ http://floradobrasil.jbrj.gov.br/>. 07 May. 2015

Lopes S, Poser GL, Kerber VA, et al. 2004. Taxonomic significance of alkaloids and iridoid glucosides in the tribe psychotrieae (Rubiaceae). Biochemical Systematics and Ecology 32: 1187-1195.

Luchi AE. 1990. Estudo anatômico do lenho em espécies de mata ciliar da Serra do Cipó (MG). Msc Thesis, Universidade de São Paulo, Brazil.

Manão CYG. 2011. Composição florística e estrutura da comunidade arbustivo-arbórea de um trecho de floresta submontana na vertente sudeste do Parque Estadual da Ilha Grande, Angra dos Reis / RJ. Msc Thesis, Universidade do Estado do Rio de Janeiro, Brazil.

Nakata PA. 2012. Engineering Calcium Oxalate Crystal Formation in Arabidopsis. Plant and Cell Physiology 53: 1275-1282.

Nepokroeff M, Bremer B, Sytsma KJ. 1999. Reorganization of the genus Psychotria and tribe Psychotrieae (Rubiaceae) inferred from ITS and $r b c \mathrm{~L}$ sequence data. Systematic Botany 24: 5-27.

Oliveira RR, Netto ALC 2001. Captura de nutrientes atmosféricos pela vegetação na Ilha Grande, RJ. Pesquisa Botânica 51: 31-49.

Rogers GK. 1981. The wood anatomy of Gleasonia, Henriquezia, and Platycarpum (Rubiaceae) and its bearing on their classification: some new considerations. Brittonia 33: 461-465.

Rogers GK. 1984. Gleasonia, Henriquezia, and Platycarpum (Rubiaceae). New York, Flora Neotropica monograph 39.

Sano Y. 2004. Intervascular pitting across the annual ring boundary in Betula platyphylla var. japonica and Fraxinus mandshurica var. japonica. IAWA Journal 25: 129-140. 
Silva Neto SJ, Peixoto A. 2012. Rubiaceae do Parque Nacional do Itatiaia Rio de Janeiro, Brasil. Boletim de Pesquisa do PNI 14: 1-139.

Sokal RR. 1986. Spatial data analysis and historical processes. In: Diday E, Escoufier Y, Lebart L, Pagès J, Schektman Y, Tomassone R. (eds.) Data Analysis and Informatics IV. North-Holland- Amsterdam, Elsevier Publishers.

Sperry JS, Nichols KL, Sullivan JE, Eastlack SE. 1994. Xylem embolism in ring-porous, diffuse-porous, and coniferous trees of northern Utah and interior Alaska. Ecology 75: 1736-1752.

StatSoft. 1993. STATISTICA. Tulsa, StatSoft.

Taylor CM. 1996. Overview of the Psychotrieae (Rubiaceae) in the Neotropics. Opera Botanica Belgica 7: 261-270.
Taylor CM. 2007. Psychotria L. In: Wanderly MGL, Shepherd GJ, Giulietti AM. (eds.) Fl. Fanerog. Estado São Paulo. São Paulo, Editora Hucitec. 5: 389-412.

Ter Welle BJH, Loureiro AA, Lisboa PLB, Koek-Noorman J. 1983. Systematic wood anatomy of the tribe Guettardeae (Rubiaceae). Botanical Journal of the Linnean Society 87: 13-28.

Tyree MT, Sperry JS. 1989. Vulnerability of xylem to cavitation and embolism. Annual Review of Plant Physiology and Molecular Biology 40: 19-38.

Williams J, León H. 2005. Anatomía ecológica del xilema secundário de un bosque seco tropical de Venezuela. Acta Botánica Venezuelica, 28: $1-22$.

Zar JH. 1996. Bioestatistical Analysis. 3rd. edn. New Jersey, Prentice Hall. 